\title{
Non-exponential tunneling and control of microwave absorption lineshapes via Fano resonance for electrons on Helium
}

\author{
Solomon Duki and Harsh Mathur \\ Department of Physics, Case Western Reserve University, \\ 10900 Euclid Avenue, Cleveland OH 44106-7079
}

\begin{abstract}
We consider the application of a small in-plane magnetic field to electrons on a helium surface in a perpendicular magnetic field. Certain states that were bound to the helium surface then dissolve into the continuum turning into long-lived resonances. As a result microwave absorption lines acquire an asymmetric Fano lineshape that is tunable by varying the microwave polarisation or the in-plane magnetic field. Electrons trapped in a formerly bound state will tunnel off the surface of helium; we show that under suitable circumstances this "radioactive decay" can show damped oscillations rather than a simple exponential decay. The mechanism for oscillatory exponential decay is not specific to electrons on Helium and this effect may also be relevant elsewhere in physics.
\end{abstract}

Electrons deposited on the surface of a pool of liquid helium form a high mobility two-dimensional electron gas that has been used to study fundamental problems of condensed matter physics including electron localisation and Wigner crystallisation [1]. Recently there has been interest in using this system to build a quantum computer due to its high degree of quantum coherence 2. In this design it is envisaged that quantum computing protocols will be implemented by driving transitions between electronic subband states using microwaves.

The purpose of this Letter is to show that in the presence of a strong magnetic field, tilted with respect to the helium surface, the shape of some microwave absorption lines is determined by Fano resonance effects [3]. This allows the lineshape to be precisely controlled by varying the magnetic field and microwave polarisation. Apart from any practical relevance, using quantum interference to control lineshapes is also of fundamental interest. For example, electromagnetically induced transparency is a quantum interference effect that alters lineshapes [4; its implementation in Bose condensates has led to fascinating phenomena, such as an extreme slowing down of light [5].

The key physics that underlies the effects discussed here is that in a perpendicular magnetic field it is possible for certain electronic states that are bound to the surface of helium to be nevertheless degenerate with unbound continuum states. When the the magnetic field is tilted, these bound states dissolve into the continuum leaving behind long-lived resonances. Thus electrons that occupy the formerly bound states will eventually escape the surface of helium via quantum tunneling. Remarkably we find that under suitable conditions this "radioactive decay" can be nonexponential in its time dependence: the probability that the electron is still bound can undergo (damped) oscillations, again due to quantum interference. Such non-exponential tunneling decay may be relevant to other areas of physics since the mechanism is not unique to electrons on helium. Indeed there is interest in non-exponential quantum tunneling as far afield as cosmology where there has been a recent analysis of departures from exponential behaviour in the long-time limit [6. The effect analysed here applies even in the short-time limit. Another more formal result derived in this Letter is that the dissolved bound state leaves its imprint on the scattering phase shift of the continuum states; namely, the phase shift jumps by $\pi$ as the energy is swept through the (renormalised) energy of the former bound state.

Before presenting our results it is useful to review Fano's analysis [3] of a single bound state $|b\rangle$ that is coupled by a perturbation $V$ to a continuum of states $|E\rangle$. The bound and the continuum states are taken to be eigenstates of an unperturbed Hamiltonian, $H_{0}$, with eigenvalues $\varepsilon_{b}$ and $E$ respectively. It is assumed that the bound state is normalised, $\langle b \mid b\rangle=1$, and orthogonal to the continuum states, $\langle b \mid E\rangle=0$, which in turn are mutually orthonormal, $\left\langle E \mid E^{\prime}\right\rangle=\delta\left(E-E^{\prime}\right)$. The perturbation couples the bound state to the continuum states via the matrix element $\langle E|V| b\rangle=V(E)$; other matrix elements of the perturbation are presumed to vanish $\left[\left\langle E|V| E^{\prime}\right\rangle=\langle b|V| b\rangle=0\right]$. As a result of the perturbation the bound state dissolves and the full Hamiltonian $H_{0}+V$ only has continuum eigenstates, denoted $\left|\psi_{E}\right\rangle$, with continuous eigenvalue $E$. Fano's original evaluation of the perturbed eigenstates $\left|\psi_{E}\right\rangle$ made use of arcane delta function identities but in fact the same results may be derived by observing that the state $\left|\psi_{E}\right\rangle$ must obey the familiar LipmannSchwinger equation,

$$
\left|\psi_{E}\right\rangle=|E\rangle+\left(E-H_{0}\right)^{-1} V\left|\psi_{E}\right\rangle,
$$

which can be solved iteratively to obtain the overlap

$$
\left\langle b \mid \psi_{E}\right\rangle=\frac{1}{\pi V(E)} \sin [\Delta(E)]
$$


and a more complicated expression for $\left\langle E^{\prime} \mid \psi_{E}\right\rangle$ 9. Together these overlaps fully determine the perturbed eigenstate $\left|\psi_{E}\right\rangle$. Here the phase angle $\Delta(E)$ is defined via

$$
\tan \Delta(E)=\frac{\pi|V(E)|^{2}}{E-\varepsilon_{b}-F(E)}
$$

and $F(E)$ is the real part of the bound state self-energy

$$
F(E)=P \int d E^{\prime}\left|V\left(E^{\prime}\right)\right|^{2} \frac{1}{E-E^{\prime}} .
$$

To the extent that we may assume $F(E)$ and $V(E)$ are slowly varying, $\left|\left\langle b \mid \psi_{E}\right\rangle\right|^{2}$ is a sharply peaked Lorentzian centred at $\epsilon_{b}$, the renormalised value of the bound state energy (defined as the energy at which $E-\varepsilon_{b}-F(E)$ vanishes). The phase $\Delta(E)$ varies monotonically from $\pi$ to zero as $E$ varies from well below the renormalised energy, $\epsilon_{b}$, to well above; it equals $\pi / 2$ right at $\epsilon_{b}$.

Now suppose a time-dependent perturbation $T$ drives a transition between some additional state $|i\rangle$ and the bound state $|b\rangle$ and the continuum states $|E\rangle$. A simple calculation using Fermi's golden rule shows that the transition rate to an exact eigenstate of energy $E$ (normalised to the rate into the corresponding unperturbed state) is given by

$$
\frac{\left|\left\langle\psi_{E}|T| i\right\rangle\right|^{2}}{|\langle E|T| i\rangle|^{2}}=\frac{|q+\epsilon|^{2}}{1+\epsilon^{2}} \text {. }
$$

Here the asymmetry parameter

$$
q=\frac{1}{\pi V(E)^{*}} \frac{\langle b|T| i\rangle}{\langle E|T| i\rangle}
$$

compares the relative couplings to the bound and unperturbed continuum states and $\epsilon=\cot \Delta(E)$ is a suitably normalised measure of the distance in energy from location of the Fano resonance, $\epsilon_{b}$. Eq $(6)$ is the celebrated lineshape derived by Fano. It shows that upon adding the perturbation $V$, although the bound state dissolves into the continuum, it leaves behind a trace in the form of a sharp absorption feature in transitions to the perturbed continuum. The Fano lineshape is asymmetric for generic $q$; it reduces to the familiar symmetric Lorentzian or Breit-Wigner form only in the limit $q \rightarrow \infty$.

We turn now to the mapping between electrons on helium and Fano's model. We take the helium surface to lie in the $y-z$ plane. The electrons are bound to the surface by a potential $V(x)=-Q e / 4 \pi \epsilon_{0} x+e F x$ for $x>0$. We assume that electrons cannot penetrate the helium and apply a hardwall boundary condition at $x=0$. Here $e$ is the magnitude of the electron charge and $Q=7 \times 10^{-3} e$ is the magnitude of the

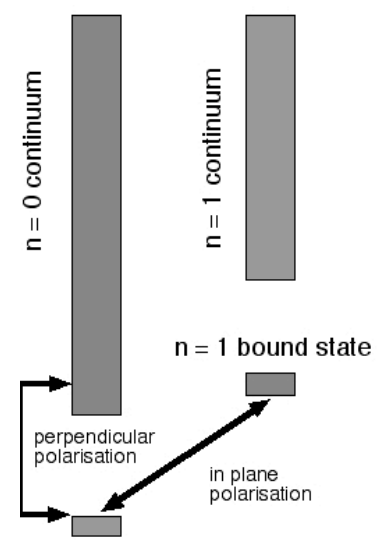

$\mathrm{n}=0$ bound state (a)

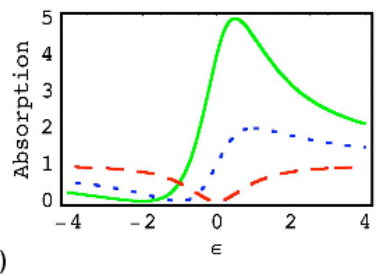

(b)

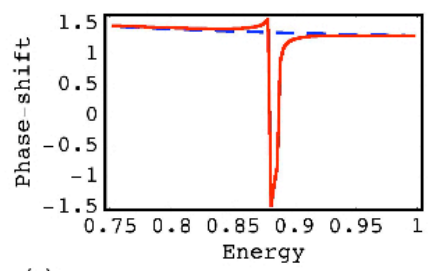

(c)
FIG. 1: (a) Schematic energy level diagram for electrons on helium in a perpendicular magnetic field. For simplicity only the lowest bound state and the continuum states are shown for the $n=0$ and $n=1$ Landau levels. Also shown are the polarisations of microwave radiation needed to drive transitions from the $n=0$ bound state to the $n=0$ continuum and the $n=1$ bound state. (b) The Fano lineshape for asymmetry parameter $q=0$ (dashed red curve), $q=1$ (dotted blue curve) and $q=2$ (solid green curve). (c) The phase shift for perturbed continuum states (solid red curve) calculated by numerical solution of the full Schrödinger equation shows the jump of $\pi$ relative to the unperturbed phase shift (dotted blue curve) as predicted by the approximate truncation to Fano's model.

image charge that forms in the dielectric helium. $F$ is an additional holding field that may be applied. In addition the magnetic field is given by $B_{x}=B_{\perp}, B_{y}=$ 0 and $B_{z}=B_{\|}$. We adopt the gauge $A_{x}=-B_{\|} y, A_{y}=$ 0 and $A_{z}=B_{\perp} y$. It will be convenient to work in units where $e B_{\perp}=\hbar=m=1$ where $m$ is the electron mass. The case that the inplane field vanishes corresponds to the unperturbed problem in Fano's model. In this case the Schrödinger equation is separable and has solutions of the form $\exp (i p z) \varphi_{n}(y-p) \xi(x)$ where $\varphi_{n}$ denotes the $\mathrm{n}^{\text {th }}$ eigenfunction of a one dimensional harmonic oscillator of unit mass and frequency. The $x$ motion is governed by the one-dimensional equation

$$
-\frac{1}{2} \frac{\partial^{2}}{\partial x^{2}} \xi+V(x) \xi=\left[E-\left(n+\frac{1}{2}\right)\right] \xi .
$$

Depending on whether the energy for $x$ motion, $k^{2} / 2=$ $E-(n+1 / 2)$, is negative or positive the wavefunction will be a bound subband wavefunction $\xi_{\nu}(x)$ or a scattering state $\xi_{\mathrm{sc}}(x, k)$. In the absence of a holding field $F$ the bound wavefunctions have the same form as the s-wave states of hydrogen and a Bohr spectrum $\varepsilon_{\nu}=-R / \nu^{2}$ where $\nu=1,2,3, \ldots$ is the subband index of the bound state and $R \approx 7.6 \mathrm{~K}$. The scattering states 
have the asymptotic form

$\xi_{\mathrm{sc}}(x, k) \rightarrow \frac{1}{\sqrt{2 \pi k}}(\exp [-i k x-i \delta(k)]+\exp [i k x+\delta(k)])$

as $x \rightarrow \infty$. By virtue of the hard wall boundary condition the reflected amplitude has unit magnitude and the scattered state is fully specified by the single phase shift $\delta(k)$ between the incoming and outgoing states. In summary the eigenstates may be written in the form $|p, n, \nu\rangle$ for the bound subband states and $|p, n, k(\mathrm{sc})\rangle$ for the unbound scattering states. The energy of the state is given by $E=(n+1 / 2)+\varepsilon_{\nu}$ for the bound states and $E=(n+1 / 2)+k^{2} / 2$ for the continuum. Note that the energy is independent of $p$ reflecting the massive Landau degeneracy of these subband states.

Fig 1 shows these energy levels schematically. From this figure we see that for sufficiently large perpendicular field (of order $6 \mathrm{~T}$ ) the lowest bound state in the $n=1$ Landau level can be degenerate with the unbound continuum states of the $n=0$ Landau level. If we now turn on a small inplane magnetic field the most important effect of this perturbation on the bound state is to couple it into the $n=0$ continuum 10]. In the same way that an atom driven near resonance may be truncated into a two-level system, here we may truncate the Hilbert space to just the $n=1$ bound state and the $n=0$ continuum. This truncation maps the problem to Fano's model: the lowest bound $n=1$ subband state is identified with $|b\rangle$ and the $n=0$ continuum with $|E\rangle$. In our units and gauge the perturbation is $-i\left(B_{\|} / B_{\perp}\right) y \partial / \partial x$, and the matrix element $V(E)$ in Fano's model is given by

$$
V\left(\frac{k^{2}}{2}\right)=-\frac{i}{\sqrt{2}} \frac{B_{\|}}{B_{\perp}} \int_{0}^{\infty} d x \xi_{b}(x) \frac{\partial}{\partial x} \xi_{\mathrm{sc}}(x, k)
$$

Having mapped the problem to Fano's model we now consider microwave absorption. For the unperturbed problem with a perpendicular magnetic field it is easy to show that radiation that is polarised in the plane couples a state $|p, n, \nu\rangle$ to states with the same $p$ and subband state and Landau index $n \pm 1$. Radiation polarised perpendicular to the plane leaves $p$ and the Landau index unchanged but can cause transitions between the subbands or between a bound subband and a continuum state. More explicitly the transition matrix element for radiation polarised in the plane is

$\left\langle p^{\prime} n^{\prime} \nu^{\prime}\left|T_{\|}\right| p n \nu\right\rangle=i \delta\left(p-p^{\prime}\right) \frac{1}{\sqrt{2}} \frac{\Omega_{E}}{\omega} \delta_{\nu^{\prime} \nu}\left(\delta_{n^{\prime}, n+1}+\delta_{n^{\prime}, n-1}\right)$.

Here $\Omega_{E}=e \mathcal{E} /\left(\sqrt{\hbar e B_{\perp}}\right)$ where $\mathcal{E}$ is the magnitude of the oscillating electric field and $\omega$ is its frequency. Similarly the transition matrix element for radiation polarised perpendicular to the plane is

$$
\left\langle p^{\prime} n^{\prime} \nu^{\prime}\left|T_{\perp}\right| p n \nu\right\rangle=i \delta\left(p-p^{\prime}\right) \frac{\Omega_{E}}{\omega} \delta_{n^{\prime} n} \int_{0}^{\infty} d x \xi_{\nu^{\prime}}^{*}(x) \frac{\partial}{\partial x} \xi_{\nu}(x) .
$$

Now if we take the lowest subband state in the $n=0$ Landau level as our state $|i\rangle$ evidently it is coupled to the state $|b\rangle$ (lowest subband state in the $n=1$ Landau level) by microwaves that are polarised in the plane; it is coupled to the continuum $|E\rangle$ (unbound states in the $n=0$ Landau level) by microwaves polarised perpendicular to the plane. Microwaves with intermediate polarisation will couple to both bound state and continuum with a relative strength that is tunable by varying the polarisation. It follows that in the presence of a perturbing inplane magnetic field the microwave absorption will show a Fano resonance with an asymmetry parameter $q$ that can be tuned by varying polarisation from zero (pure perpendicular polarisation) to $\infty$ (pure in-plane polarisation).

Another consequence of our mapping to Fano's model is that when the inplane perturbation is turned on the bound state $|b\rangle$ will dissolve into the continuum $|E\rangle$ yielding the perturbed eigenstates $\left|\psi_{E}\right\rangle$. Using Fano's expression for the overlap of $\left|\psi_{E}\right\rangle$ with $|E\rangle$ we find by straightforward asymptotic integration that the wavefunctions of the corresponding eigenstates have the $x \rightarrow \infty$ asymptotic behaviour

$$
\begin{gathered}
\frac{1}{\sqrt{2 \pi k}} \exp (i p z) \phi_{0}(y-p)(\exp [-i k x-i \delta(k)-i \Delta] \\
+\exp [i k x+i \delta(k)+i \Delta]) .
\end{gathered}
$$

Comparing to the asymptotic $x$ dependence of the unperturbed states $|E\rangle$, eq (8), we arrive at the elegant conclusion that although the bound state dissolves into the continuum it leaves behind an imprint in the perturbed continuum states in the form of an extra phase shift $\Delta$. As noted above the phase $\Delta$ jumps abruptly by $\pi$ as we sweep through the renormalised energy of the formerly bound state.

We have derived this phase shift within the mapping to Fano's model. Since the prediction does not depend on the specific form of the binding potential $V(x)$ we can check our prediction, and the veracity of the mapping to Fano's model, by numerically solving the Schrödinger equation for the case that the confining potential is a rectangular well, a circumstance that may be efficiently solved by the numerical methods of Nöckel and Stone [7]. Fig 1 shows that the expected phase jump indeed occurs without making the truncation to Fano's model.

Finally we turn to non-exponential tunneling decay. As a prelude consider the basic Fano model of a single bound state coupled by a perturbation to a continuum. 
If the system starts in the bound state initially, the amplitude to remain in the bound state at time $t$ is

$$
\begin{aligned}
b(t) & =\int d E\left|\left\langle b \mid \psi_{E}\right\rangle\right|^{2} \exp [-i E t] \\
& \approx \exp \left(-i \epsilon_{b} t\right) \exp \left(-\frac{t}{2 \tau_{b}}\right) .
\end{aligned}
$$

Here $\epsilon_{b}$ is the renormalised bound state energy defined below eq (4) and $\tau_{b}$, the lifetime of the bound state, is $\left|2 \pi V\left(\epsilon_{b}\right)\right|^{-1}$. The oscillatory exponential behaviour of $b(t)$ is because the amplitude $\left|\left\langle b \mid \psi_{E}\right\rangle\right|^{2}$ is a Lorentzian sharply peaked about the renormalised energy. The probability to remain in the bound state is therefore a pure exponential decay, $|b(t)|^{2}=\exp \left(-t / \tau_{b}\right)$. This is the usual reason that tunneling decay is exponential.

Now consider instead a model in which two or more bound states are coupled to the same continuum by a perturbation. Even if the perturbation does not directly couple the two bound states, they become effectively coupled due to their coupling to the same continuum. Thus if the system starts in one bound state it will undergo damped oscillations into the other bound state(s), the damping being produced by the tunneling decay into the continuum.

To put this idea on a quantitative footing consider, again following Fano [3], a model with $n$ bound states $|i\rangle$ where $i=1,2,3, \ldots n$ and a single continuum $|E\rangle$. These states are assumed to be orthonormal and eigenstates of the unperturbed Hamiltonian $H_{0}$ with eigenvalues $\varepsilon_{i}$ and $E$ respectively. We assume that the only non-vanishing matrix elements of the perturbation are $\langle E|V| i\rangle=V_{i}(E)$. Once the perturbation is turned on the bound states will dissolve into the continuum; the perturbed continuum states will be denoted $\left|\psi_{E}\right\rangle$. Once again these states are most easily derived using the Lipmann-Schwinger method. A key quantity in this analysis is the hermitean part of the bound state self-energy matrix [1]

$$
F_{i j}(E)=P \int d E^{\prime} \frac{V_{i}\left(E^{\prime}\right) V_{j}^{*}\left(E^{\prime}\right)}{E-E^{\prime}} .
$$

Formally the oscillations in the tunneling may be traced to the fact that the self-energy term $F_{i j}$ is not diagonal. It is useful to determine the eigenvectors of the $n \times n$ matrix $\varepsilon_{i} \delta_{i j}+F_{i j}(E)$ denoted as $A_{i \nu}(E)$ with eigenvalues $\varepsilon_{\nu}$. If we define $V_{\nu}(E)=\sum_{i} V_{i}(E) A_{i \nu}(E)$ then we find that the overlap of the perturbed continuum eigenstates $\left|\psi_{E}\right\rangle$ with the unperturbed bound states is

$$
\sum_{i=1}^{n} A_{i \mu}^{*}(E)\left\langle i \mid \psi_{E}\right\rangle=\frac{1}{\pi V_{\mu}(E)} \tan \left[\Delta_{\mu}(E)\right] \cos [\Delta(E)] .
$$

Here the phase shifts $\Delta_{\mu}$ and $\Delta$ are defined via

$$
\tan \Delta_{\mu}(E)=\frac{\pi\left|V_{\mu}(E)\right|^{2}}{E-\varepsilon_{\mu}(E)} ; \quad \tan \Delta=\sum_{\mu=1}^{n} \tan \Delta_{\mu} .
$$

Once again the renormalised energies $\epsilon_{\mu}$ may be defined as the values of $E$ at which $E-\varepsilon_{\mu}(E)$ vanishes. The phase $\Delta_{\mu}$ then jumps from $\pi$ to zero as the energy is swept through $\epsilon_{\mu}$ from below.

Fano studied the lineshape of radiative transitions into a continuum coupled to multiple bound states using the model and solution outlined above. Here we investigate what happens if the system starts in one of the bound states $|i\rangle$. Making the same approximations that led to eq (13) a straightforward calculation reveals that the amplitude to remain in the state $|i\rangle$ is

$$
b_{i}(t)=\sum_{\mu}\left|A_{i \mu}\left(\epsilon_{\mu}\right)\right|^{2} \exp \left(-i \epsilon_{\mu} t\right) \exp \left(-\frac{t}{2 \tau_{\mu}}\right) \text {. }
$$

Here $\tau_{\mu}=2 \pi\left|V_{\mu}\left(\epsilon_{\mu}\right)\right|$. Since the different terms in the superposition oscillate at different frequencies they will interfere leading to oscillations in the probability $\left|b_{i}(t)\right|^{2}$. For example if there are just two coupled bound states

$$
\begin{aligned}
\left|b_{1}(t)\right|^{2} & =\left|A_{11}\left(\epsilon_{1}\right)\right|^{4} \exp \left(-\frac{t}{\tau_{1}}\right)+\left|A_{12}\left(\epsilon_{2}\right)\right|^{4} \exp \left(-\frac{t}{\tau_{2}}\right) \\
& +2\left|A_{11}\right|^{2}\left|A_{12}\right|^{2} \cos \left[\left(\epsilon_{n}-\epsilon_{m}\right)\right] \exp \left(-\frac{t}{2}\left[\frac{1}{\tau_{1}}+\frac{1}{\tau_{2}}\right]\right) .
\end{aligned}
$$

If the decay times are comparable and the oscillation frequency sufficiently high a departure from simple exponential decay should be easily observable.

Evidently this analysis is relevant to electrons on helium in a tilted magnetic field where there are in fact multiple bound states that couple to the same continuum. There are already experimental studies on the tunneling rates of electrons on helium in a magnetic field [8] but not from the type of resonant states considered here. It would be desirable in future work to identify an optimum set of coupled bound states for an experimental detection of oscillatory exponential decay. In addition analogues throughout atomic, solid state, nuclear and particle physics bear investigation. Another important extension of this work is to consider the damping of Fano resonant effects by a bath of oscillators. Such damping is interesting both as a matter of principle and in order to incorporate the effect of ripplons and vapour scattering on the single particle effects analysed here.

In summary we have shown that electrons on the liquid helium in a magnetic field have bound states that 
dissolve into the continuum when an inplane magnetic field is applied; however they leave behind an imprint in the form of a phase shift and a sharp microwave absorption feature whose lineshape is tunable by varying the polarisation and the inplane magnetic field. In addition we point out that the tunneling decay of electrons from these formerly bound states may have an oscillatory exponential form.

We acknowledge discussions with Arnold Dahm and Francesc Ferrer.

[1] E. Andrei (ed), Two Dimensional Electron Systems on Helium and Other Cryogenic Substrates (Kluwer Academic, London, 1997).

[2] P.M. Platzman and M.I. Dykman, Science 284, 1967 (1999).

[3] U. Fano, Phys Rev 124, 1866 (1961).

[4] M. Fleischhauer, A. Imamoglu and J.P. Marangos, Rev Mod Phys 77, 633, (2005).
[5] L.V. Hau, S.E. Harris, Z. Dutton and C.H. Behroozi, Nature 397, 594 (1999).

[6] L.M. Krauss and J. Dent, hep-ph/arXiv:0711.1821; to appear in Phys Rev Lett.

[7] J.U. Nöckel, Phys Rev B45, 14225 (1992); J. U. Nöckel and A.D. Stone, Phys Rev B51, 17219 (1995).

[8] L. Menna, S. Yücel and E.Y. Andrei, Phys Rev Lett 70, 2154 (1993).

[9] Some details: The first-order solution to eq (1) is obtained by replacing the perturbed eigenstate $\left|\psi_{E}\right\rangle$ on the right hand side with the unperturbed state $|E\rangle$; the second-order solution, by replacing $\left|\psi_{E}\right\rangle$ on the right-hand side with the first-order solution; and so on. The resulting perturbative series is geometric. Its summation is facilitated by defining the self energy $\Sigma(E)=\left\langle b\left|V\left(E-H_{0}\right)^{-1} V\right| b\right\rangle$ by analogy to Dyson's equation in field theory. By this procedure we obtain eq (2) and $\left\langle E^{\prime} \mid \psi_{E}\right\rangle=\delta\left(E-E^{\prime}\right) \cos \Delta+$ $(1 / \pi)\left[V\left(E^{\prime}\right) / V(E)\right] \sin \Delta /\left(E-E^{\prime}\right)$ in agreement with Fano's results.

[10] More precisely, the bound state with a given $p$ gets coupled to the continuum state with the same $p$ value.

[11] The self-energy $\Sigma_{i j}(E) \equiv\left\langle i\left|V(E-H)^{-1} V\right| j\right\rangle$. 